\title{
The variation in genetic material of a high Alpine catchment reveals (sub)surface exchange
}

Elvira Maechler ${ }^{1,2}$, Natalie Ceperley ${ }^{3,4}$, Anham Salyani ${ }^{3}$, Jean-Claude Walser ${ }^{5}$, Annegret Larsen ${ }^{3,6}$, Bettina Schaefli, ${ }^{3,4}$, and Florian Altermatt ${ }^{1,2}$

${ }^{1}$ Eawag: Swiss Federal Institute of Aquatic Science and Technology, Department of Aquatic Ecology, Dübendorf, Switzerland (elvira.maechler@eawag.ch)

${ }^{2}$ Institute of Evolutionary Biology and Environmental Studies, University of Zurich, Zürich, Switzerland

${ }^{3}$ Institute of Earth Surface Dynamics, Faculty of Geosciences and Environment, University of Lausanne, Lausanne,

Switzerland

${ }^{4}$ Geography Institute, University of Bern, Bern, Switzerland

${ }^{5}$ Federal Institute of Technology (ETH), Zürich, Genetic Diversity Centre, Zürich, Switzerland

${ }^{6}$ Soil Geography and Landscape Group, Wageningen University, Wageningen, The Netherlands 